\title{
Population-based data as an important contribution to better investigate chronic respiratory diseases
}

\section{Annesi-Maesano}

Monaldi Arch Chest Dis 2005; 63: 2, 69-71.

Epidemiology of Allergic and Respiratory Diseases (EPAR); INSERM, Paris; France.

Correspondence: Dr. Isabella Annesi-Maesano, INSERM U 472 Epidemiology \& Biostatistics; 16 Ave Paul Vaillant-Couturier, 94807 Villejuif Cedex, France; e-mail: annesi@vjf.inserm.fr

This issue of the Monaldi Archives for Chest Diseases provides interesting population-based data on various respiratory diseases: two reports on cross-sectional data and one on longitudinal data. On the one side there are two reports, dealing with airflow limitation and Chronic Obstructive Pulmonary Diseases (COPD) on which there have been scarce amount of data so far, on the other side is a report dealing with asthma which has been more extensively studied at the population level (see the European Community Respiratory Health Survey (ECRHS) and the International Study of Asthma and Allergies in Childhood (ISAAC)). Surveying the general population constitutes an interesting approach as asthma and other respiratory diseases are widely spread at the population level. Considering only respiratory patients could select particularly severe phenotypes of these diseases and thus reduces evidence on some potential relationships and mechanisms.

In the Asthma and Long-term Oxygen Therapy (ALOT)) study, Caramori and colleagues could not find any non-smoking asthmatics among patients in long-term domiciliary oxygen therapy (LTOT) drawn from the general population of 3 referral hospitals of Bologna and Ferrara in the North-East of Italy [1]. The departure hypothesis was that asthma would have not been a common cause of severe chronic respiratory failure necessitating LTOT due to reversibility of inflammatory lesions associated with the asthmatic condition. This might be true overall in the absence of the noxious effect of tobacco smoking, which has a certain pro-inflammatory action. Thereafter, the ALOT data supports current consensus according to which asthma and COPD are different diseases. However, at the population level, active smoking has been related in different ways to asthma [2]. Population-based data have shown that active smoking may play three different roles in asthma: risk factor, selection factor (the so called "healthy smoker effect") or effect modifier (in the case of severity or diagnosis), respectively. They have also shown that being asthmatic does not operate as a deterrent in starting to smoke but is merely an incentive to stop smoking later in life, mainly in cases of severe asthma. Young people and females (gender issue was not examined in the presented data due to the reduced sample size) seem more susceptible to the effects of active smoking with respect to asthma. In addition, different types of asthma have been described according to the timing of smoking onset in a population-based sample [3]. Asthma either never accompanied by smoking or followed by smoking onset might be characterised by an allergic pattern. Asthma occurring after smoking has no allergic component and is related only to $\mathrm{FEV}_{1}$ decline. Whether there is a contradiction between previous population-based data and the ALOT study cannot be exhaustively stated from the existing data. Asthmatic patients could be assigned less frequently to LTOT than COPD patients, which would have prevented the possibility of finding asthmatics in the population recruited in the three hospitals. It cannot be excluded that they could have suffered from asthma before the onset of COPD. However, no past history of asthma and/or acute wheezing episodes was reported by the ALOT patients according to the clinical records. More importantly, observational data are consistent with experimental data: asthma and COPD inflammations differ in many respects [4]. It is likely that ALOT data concerns an older population and a different asthma phenotype than those considered in previous population-based studies. Longitudinal studies are warranted to further clarify the relationships among asthma phenotypes according to the sequence of disease onset and smoking. In the meantime, the ALOT study has provided the opportunity to investigate mechanisms underlying severe chronic respiratory failure, which is useful in view of the management and the prevention of such a condition.

A necessary confirmation that smoking cessation is urged has been provided by the Riccioni and colleagues' article [5]. From January to December 2000,1514 consecutive individuals aged $\geq 25$ years underwent spirometry and bronchial reversibility test with salbutamol. Individuals with bronchial asthma, bronchiectasis, and interstitial lung disease, positive reversibility to bronchial test and insufficient compliance to the execution of the test were excluded from the study. The prevalence of bronchial obstruction varied according to the 
criteria used: $27.5 \%$ (ERS), 33\% (GOLD) and $47.3 \%$ (ATS). The prevalence increased with age and was higher as predicted but scarcely documented taking the 3 different criteria into account in smokers and former smokers. In a previous Italian survey conducted among 4,353 adults $(<75$ years) drawn from the general population of 2 contrasted areas, one rural (Po delta) and the other urban (Pisa), the prevalence of chronic airflow limitation varied from $29 \%$ (rural zone) to $25 \%$ (urban zone) for asthma, from 32 (rural zone) to $39 \%$ (urban zone) for chronic bronchitis and from $40 \%$ (rural zone) to $28 \%$ (urban zone) for emphysema. A large proportion of the population displayed chronic airflow obstruction in the absence of a respiratory disease. Thereafter, Riccioni et al's statistics confirm in line with previous works the high prevalence of airway obstruction at the population level not only in current and former smokers but also in non-smokers $21 \%$ according to ERS criteria, $24.9 \%$ according to GOLD criteria and $38.6 \%$ according to ATS criteria). Such studies respond to a need for a better estimation of the under-diagnosis of airflow obstructions and COPD, which is becoming extremely noticeable at the population level. In this context, the data by Riccioni and colleagues provides a useful insight into the different degree of airflow obstruction; mild and moderate airflow obstruction being less diagnosed than the other degrees of the conditions. Yet, it has been proven that preventive measure should address initial degrees of the condition in order to be effective.

A direct issue raised by this work is about the use of pre-bronchodilator $\mathrm{FEV}_{1} / \mathrm{FVC}$ values for the definition of airflow limitation, as this leads to potential over-diagnosis and over-estimation of the severity of this disease, which may be particularly important to young adults in whom reversible airflow limitation may not be uncommon. Sterk has extensively discussed this point in a recent issue of the European Respiratory Journal (6). Are we on the right track, asks the ERJ Editor, by disseminating information on the prevalence and severity of COPD in the absence of measuring post-bronchodilator spirometry into the public domain? The ATS/ERS standards for the diagnosis and treatment of patients with COPD (7) clearly sticks to the GOLD criteria based on post-bronchodilator $\mathrm{FEV}_{1} / \mathrm{FVC}$ and $\mathrm{FEV}_{1}$.

The last report presents new longitudinal data on the evolution of asthma prevalence between 1994 and 1999 in the town of Turin [8]. Asthma prevalence was assessed in 3 schools using the same protocol 5 years apart. Just as in previous studies conducted in industrialised countries, asthma prevalence did not increase in the surveyed schools of Turin between 1994-5 and 1998-9 for a total of almost 500 children. The authors advocated as an explanation of the observed phenomenon the fact that in the same period the exposure to potential risk factors for asthma has also be stationary or has even reduced. Indeed, exposure to parental tobacco smoke and to outdoor air pollution equally assessed during the 2 surveys seem to have diminished although not significantly. So far, few studies have reported on risk factors assessed simultaneously as health outcomes. As a consequence of the diminished exposure to environmental aggressions, the expression of the disease which is genetically determined could have been blocked. Another explanation provided by the authors for the observed stable plateau is that there has been in the meantime an increase in the sensitivity of the clinicians towards to asthma. This latter explanation seems more convincing. However, further studies are needed as the sample was small and as a consequence there was a low study power, which may be partly responsible for the lack of statistical significance of some the associations.

Asthma prevalence has incessantly increased in the past three decades. However, the latest data obtained in population-based settings in Italy as well as elsewhere have shown that prevalence has reached a stable plateau [9-11]. To evaluate whether this phenomenon is real, it is important to compare data obtained in a standardised manner in two different periods of time. This has been done in the most appropriate manner by Migliore et al., as exactly the same schools were considered after 5 years of delay. This has contributed to reduce potential bias, such as confounding related to climate, social class, diet, etc. which were avoided by taking the same schools into account.

Among 11168 individuals of 14 European countries of the ECRHS, no significant increase in the prevalence of asthma and asthma-like symptoms was observed [12]. On the contrary, the prevalence in the use of asthma medication increased significantly of $2.1 \%$ (95\% CI: 1.6 to $2.6 \%$ ). Unfortunately, the available data does not allow understanding whether such stability is due to an improvement in asthma management, which is one of the hypotheses raised by Migliore et al. Asthma prevalence continues to increase in developing countries [13-15] and this support the alternative hypothesis that in the industrialised countries asthma has stopped increasing because the genetic potential cannot express anymore of the absence of structural changes in environmental factors. But this hypothesis is difficult to test. Other studies are therefore needed.

All together these articles, although dealing with different topics and contexts, stress the need for standardised methods in order to make comparisons among data possible. This standardisation is indispensable not only in epidemiological but also in clinical settings.

\section{References}

1. Caramori G, Fabbri M, Paioli D, et al. Asthma is not a common cause of severe chronic respiratory failure in non-smokers: ALOT study. Monaldi Arch Chest Dis 2005; 63: 84-87.

2. Viegi G, Pedreschi M, Pistelli F, et al. Prevalence of airway obstruction in a general population sample/ European Respiratory Society vs. American Thoracic Society definition. Chest 2000; 117: 339s-345s.

3. Raherison C, Baldi I, Tunon-De-Lara JM, Taytard A, 
Annesi-Maesano I. Asthma phenotypes according to the timing of smoking onset in young adults. Int $J$ Tuberc Lung Dis 2003; 7 (1): 84 -92.

4. Bousquet J, Jeffery PK, Busse WW, Johnson M, Vignola AM. Asthma. From bronchoconstriction to airways inflammation and remodelling. Am J respire Crit Care Med 2000; 161: 1720-45.

5. Riccioni G, De Benedictis M, Della Vecchia R, et al. Prevalence and severity of airway obstruction in an Italian adult population. Monaldi Arch Chest Dis 2005; 63: 88-92.

6. PJ Sterk. Let's not forget: the GOLD criteria for COPD are based on post-bronchodilator $\mathrm{FEV}_{1}$. Eur Respir $J$ 2004; 23: 497-498.

7. Celli BR, MacNee W; ATS/ERS Task Force. Standards for the diagnosis and treatment of patients with COPD: a summary of the ATS/ERS position paper. Eur Respir $J$ 2004; 23: 932-46.

8. Migliore E, Piccioni P, Garrone G, et al. Changing prevalence of asthma in Turin school children between 1994 and 1999. Monaldi Arch Chest Dis 2005; 63: 74-78.

9. Maziak W, Behrens T, Brasky TM, et al. Are asthma and allergies in children and adolescents increasing?
Results from ISAAC phase I and phase III surveys in Munster, Germany. Allergy 2003; 58: 572-9.

10. Wong GW, Leung TF, Ko FW, et al. Declining asthma prevalence in Hong Kong Chinese schoolchildren. Clin Exp Allergy 2004; 34: 1550-5.

11. Garcia-Marcos L, Quiros AB, Hernandez GG, et al. Stabilization of asthma prevalence among adolescents and increase among schoolchildren (ISAAC phases I and III) in Spain. Allergy 2004; 59: 1301-7.

12. Chinn S, Jarvis D, Burney $\mathrm{P}$, et al. Increase in diagnosed asthma but not in symptoms in the European Community Respiratory Health Survey. Thorax 2004; 59: 646-51.

13. Wang XS, Tan TN, Shek LP, et al. The prevalence of asthma and allergies in Singapore; data from two ISAAC surveys seven years apart. Arch Dis Child 2004; 89: 423-6.

14. Hong SJ, Lee MS, Sohn MH, et al. Korean ISAAC Study Group. Self-reported prevalence and risk factors of asthma among Korean adolescents: 5-year follow-up study, 1995-2000. Clin Exp Allergy 2004; 34: 1556-62.

15. Banac S, Tomulic KL, Ahel V, et al. Prevalence of asthma and allergic diseases in Croatian children is increasing: survey study. Croat Med J 2004; 45: 721-6.

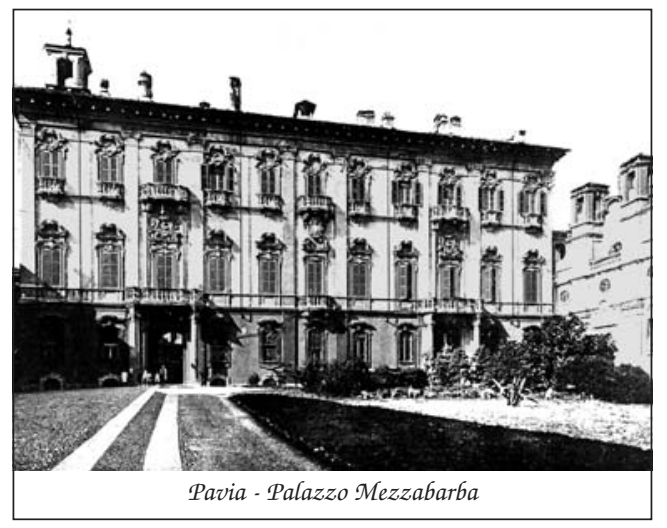

